\title{
Rheological Study of Genipin Cross-Linked Chitosan Hydrogels
}

\author{
M. José Moura, ${ }^{*, \dagger}$ M. Margarida Figueiredo, ${ }^{\ddagger}$ and M. Helena Gil ${ }^{\ddagger}$ \\ Departamento de Engenharia Química, Instituto Superior de Engenharia de Coimbra, R. Pedro Nunes, \\ 3030-199 Coimbra, Portugal, and Departamento de Engenharia Química, Universidade de Coimbra, Pólo \\ II, Pinhal de Marrocos, 3030-290 Coimbra, Portugal
}

Received July 10, 2007; Revised Manuscript Received September 24, 2007

\begin{abstract}
This paper reports the rheological behavior of chitosan solutions that have been cross-linked with different amounts of genipin, at body temperature and physiological $\mathrm{pH}$. The effect of the cross-linker loading on the rheological properties of hydrogels has been evaluated. The oscillatory time sweep method was used to monitor the dynamic viscoelastic parameters during in situ (i.e., in the rheometer) gelation experiments, enabling the determination of the gelation time. The stress and frequency sweeps were employed to measure $G^{\prime}$ of the cured hydrogels. It was found that the solutions of chitosan cross-linked with genipin, under physiological conditions, could form relatively strong elastic gels when compared to those of pure chitosan. Moreover, the gelation time obtained from the crossover of $G^{\prime \prime}$ and $G^{\prime}$ was in excellent agreement with the value obtained from the Winter-Chambon criterion. A significant reduction on this parameter was achieved even at low genipin concentrations. This behavior suggests that these formulations are able to be produced in situ and thus constitute promising matrices for cells and bioactive molecule encapsulations.
\end{abstract}

\section{Introduction}

Chitosan, an amino-polysaccharide obtained by alkaline deacetylation of chitin, is a nontoxic, biocompatible, and biodegradable $\mathrm{pH}$-dependent cationic polymer that has recently found many applications in the biomedical field. ${ }^{1-3}$ It also has important biological properties, such as antimicrobial activity and ionic binding affinity with DNA. In addition to good film and gel forming properties, one of chitosan's most promising features is its excellent ability to produce three-dimensional scaffolds. ${ }^{2,4}$

Chitosan is typically not soluble in water, but chitosan solutions can be obtained in acidic aqueous media $(\mathrm{pH}<6)$, which protonates its amino groups, rendering the polymer positively charged and, thereby, overcoming associative forces between chains. When solubilized, chitosan can form weak intermolecular links through hydrophobic interactions between residual acetyl groups. Chitosan gels can be obtained by increasing the polymer concentration (concentration-induced gelation) or by controlling the solution $\mathrm{pH} .{ }^{5}$ Regarding the latter methodology, the addition of specific basic salts, such as glycerol-phosphate, allows the increase of the solution $\mathrm{pH}$, due to the neutralizing effect of the phosphate groups, and enables the transformation of the original solution into a temperaturecontrolled $\mathrm{pH}$-dependent matrix. Such a solution remains in liquid state below room temperature at $\mathrm{pH}$ values within the physiologically acceptable neutral range (6.8 to 7.2$)$ and turns into gel when heated at body temperature, undergoing, therefore, a heat-induced gelation. ${ }^{6-8}$ When the temperature is decreased, the chitosan gel, under certain conditions, returns to the solution state, which allows the establishment of a temperature-induced cyclic behavior.

The formation of far stronger and more extensive intermolecular associations is possible through chemical cross-linking processes (covalent bond forming), which impart additional

\footnotetext{
* Corresponding author. E-mail: mjmoura@isec.pt.

Instituto Superior de Engenharia de Coimbra.

Universidade de Coimbra.
}

valuable properties to the resulting materials. In particular, crosslinking can be used to enhance mechanical strength and chemical stability, to control aqueous permeability and solubility, and to decrease the aqueous swelling features of chitosan-based materials. ${ }^{1,9-14}$

For long-term drug delivery applications, chemically induced cross-linking under physiological conditions gives rise to materials with better performance compared to physical crosslinking, as the former produces relatively stable hydrogel networks with predictable degradation behavior. ${ }^{15}$ However, the toxicity of cross-linking agents presents a major drawback in the use of polymers as injectable matrices or as in situ-forming polymer scaffolds, because their seepage into body fluids can be harmful, even at low concentrations. ${ }^{16}$ Recently, a new crosslinking agent, genipin, has been successfully used for such a purpose. ${ }^{14}$ Genipin has been reported to bind with biological tissues ${ }^{17}$ and biopolymers, such as chitosan and gelatin, ${ }^{18-20}$ leading to covalent coupling. It works as an effective cross-linking agent for polymers containing amino groups and is much less cytotoxic than glutaraldeyde (mostly used for chitosan crosslinking), ${ }^{21}$ thus satisfying one of the requirements imposed for its application in injectable matrices. Besides, it has been reported that the genipin cross-linked chitosan membranes exhibit a slower degradation rate than their glutaraldeyde crosslinked counterpart. ${ }^{13}$ Regarding drug delivery systems, it has been shown that the addition of genipin, in situ under physiological conditions, to a formulation containing chitosan allows extending the drug release period. ${ }^{22}$

Another property that plays a key role in the design of injectable scaffolds is the solidification rate or gelation time of the precursors. As a matter of fact, the gelation time also needs to be balanced, as it should be short enough to prevent heterogeneous distribution of cells and bioactive molecules and to keep the gel highly cohesive at the injection site, but on the other hand, it should be of sufficient duration to allow for a proper surgical procedure to take place. ${ }^{23}$ Despite the relevance of this property, that is, related to the structure and composition of the formulations as well as their processing conditions, the 
works related with this parameter are rather scarce. In fact, most of the rheological studies reported in the literature are mainly concerned with the improvement of the final mechanical properties and network stability of chitosan hydrogels. ${ }^{11-14}$ Besides, most of these hydrogels involve synthetic polymers and are not produced under physiological conditions (essential to keep high cell viability and molecular bioactivity). ${ }^{23}$

The work described in this paper is a further step in the investigation of the rheological behavior of chitosan-genipin solutions and the networks subsequently formed, at body temperatures and physiological $\mathrm{pH}$ values, in order to assess their potential for use as injectable scaffolds.

Theoretical Background. Because gelation is a gradual transition from a viscoelastic liquid to a viscoelastic solid, the measurements of rheological properties, being very useful, have been performed by various research groups to characterize chitosan solutions, gels, and cross-linked networks. ${ }^{7,24-30}$

Dynamic rheometry is a powerful tool for monitoring the gelation (or cross-linking) and microstructural changes in a material. ${ }^{31}$ This is because it allows properties to be probed in "at rest" conditions without disruption of the microstructure. In addition, rheometry is an effective method for studying the curing of thermosetting polymers and for the examination of viscoelastic material functions, such as dynamic shear moduli, $G^{\prime}$ and $G^{\prime \prime}$, and loss tangent, $\tan (\delta)$, defined as follows. ${ }^{32}$

The shear elastic (or storage) modulus, $G^{\prime}$, is defined as the amplitude ratio of the component of the stress $\left(\sigma_{0}\right)$ in phase with the strain to the strain amplitude $\left(\gamma_{0}\right)$ :

$$
G^{\prime}=\sigma_{0} \cos (\delta) / \gamma_{0}
$$

where $\delta$ is the phase angle between stress and strain. Similarly, the shear viscous (or loss) modulus, $G^{\prime \prime}$, is the amplitude ratio of the component of the stress in quadrature with the strain to the strain amplitude:

$$
G^{\prime \prime}=\sigma_{0} \sin (\delta) / \gamma_{0}
$$

For a perfect solid, the stress and strain waveforms are in phase, with $\delta=0^{\circ}, G^{\prime \prime}=0$, and $G^{\prime}$ having a finite value. Thus, for a given value of $\gamma_{0}, G^{\prime}$ gives a measure of the energy that is stored elastically by the system per cycle when subjected to frequency oscillation. Also, for a perfect liquid, the stress and the strain waveforms are in quadrature: $\delta=90^{\circ}, G^{\prime}=0$, and $G^{\prime \prime}$ is finite. For such a situation, $G^{\prime \prime}$ represents a measure of the energy that is dissipated during flow per cycle of oscillation for each value of $\gamma_{0}$. It follows that for any real system, with a phase displacement lying between the two classical extremes of behavior, the relative magnitudes of the storage and loss moduli will provide information regarding the proportion of the energy input that is stored elastically and that which is dissipated during the flow over each cycle of frequency oscillation. The combination of eqs 1 and 2 provides the following useful relationship:

$$
\tan (\delta)=\frac{G^{\prime \prime}}{G^{\prime}}
$$

The loss tangent gives a measure of the ratio of the energy loss to the energy stored and indicates the overall viscoelasticity of the sample. ${ }^{28}$

The formation of polymer gels can be monitored from the development of the viscoelastic functions of the material at the gel point. The gel point is one of the more important physical parameters of the gelation process, characterized by the appearance in the reactive system of a macromolecule with an infinitely large molecular weight. ${ }^{33}$ In practice, a sudden loss of flow due to the fact the viscoelastic properties change abruptly from an initially liquid-like state to a solid-like state is the most common and conventional fingerprint to the determination of the sol-gel transition point. ${ }^{34,35}$ Consequently, the sol-gel transition can be easily determined from rheological data, because it coincides with the point at which $G^{\prime}$ intersects $G^{\prime \prime}$. However, a more general and reliable method for identifying the sol-gel transition is based on the Winter-Chambon criterion, ${ }^{36}$ where the gel point is identified as the time instant at which both $G^{\prime}$ and $G^{\prime \prime}$ follow power law behavior:

$$
G^{\prime}=k^{\prime} \omega^{n} \text { and } G^{\prime \prime}=k^{\prime \prime} \omega^{n}
$$

Here, $n$ denotes the exponent, $\omega$ is the angular frequency, $k^{\prime}$ and $k^{\prime \prime}$ are proportionality constants. The exponent $n$ is termed the relaxation exponent and can be intimately related to a material's structure. ${ }^{37}$ According to eq 4 , the loss tangent is given as:

$$
\tan (\delta)=\tan \left(\frac{n \pi}{2}\right)
$$

Equation 5 implies that $\delta$ is independent of the frequency at the gel point but is proportional to the relaxation exponent. The validity of this theory has been proven to be appropriate for describing a variety of chemically and physically cross-linked systems. $^{38}$

In the present work, oscillatory rheometry, operated in time, stress, and frequency sweep modes was used for monitoring continuously the viscoelastic properties associated with the cross-linking during the gelling process and to characterizing the mechanical properties of the chemically formed networks. Rheological measurements were used to determine the gelation time by two different approaches.

\section{Materials and Methods}

Materials. The chitosan used in the experiments was purchased from Sigma-Aldrich in a powder form, with a molecular weight of approximately $2 \times 10^{5}$ Daltons and a degree of deacetylation greater than $85 \%$. Hydrated $\beta$-glycerol-phosphate disodium salt $\left(\mathrm{C}_{3} \mathrm{H}_{7}\right.$ $\mathrm{Na}_{2} \mathrm{O}_{6} \mathrm{P} \cdot \mathrm{H}_{2} \mathrm{O} ; \mathrm{FW}=218.05$ ), used to adjust the $\mathrm{pH}$ of the chitosan solutions, was also purchased from Sigma-Aldrich. Genipin (crystallike powders, reagent grade) was supplied by Challenge Bioproducts Co., Taiwan. All other reagents and solvents used were of an analytical grade.

Sample Preparation. A solution containing $2 \mathrm{~g}$ of chitosan in 100 $\mathrm{cm}^{3}$ of solution was prepared by dissolving the chitosan powder in distilled water containing $0.5 \%$ (by volume) acetic acid at room temperature. The chitosan solution was then filtered and subsequently sonicated to remove the trapped air bubbles. The final product had a light yellow color and a pH of 5. Thereafter, a given amount of glycerolphosphate disodium salt was dissolved in distilled water and carefully added, drop by drop, under magnetic stirring, to the chitosan solution to provide a clear and homogeneous liquid solution with a concentration of $1.5 \mathrm{~g}$ of chitosan in $100 \mathrm{~cm}^{3}$ of solution, with a $\mathrm{pH}$ value of 7.0. Genipin powder was dissolved in the resulting solution to produce samples with genipin loadings of $0.05,0.10,0.15$, and $0.20 \%$ (by weight). Samples without genipin were used as a control. To evaluate the influence of the $\mathrm{pH}$ on the solution rheology, samples were also prepared in the same way without the addition of the glycerol-phosphate disodium salt. The hydrogels of different cross-linking densities were subsequently cured for $12 \mathrm{~h}$ at $37{ }^{\circ} \mathrm{C}$ and subjected to oscillatory rheometry.

Rheological Characterization. The rheological characterization of the hydrogel samples was performed in a C-VOR rheometer from Bohlin Instruments, Inc., U.S.A., fitted with a cone-and-plate geometry (cone angle of $4^{\circ}$ and diameter of $20 \mathrm{~mm}$ or $40 \mathrm{~mm}$ ) and a circulating 
environmental system for temperature control. The rheometer used is a shear stress controlled instrument that consists of applying a torque (force) and measuring the resultant displacement (movement). Torque and displacement are converted to "rheological format" by means of the measuring system constants. To prevent drying of the samples during experiments, a steel ring of $\sim 60 \mathrm{~mm}$ was placed around the measuring geometry, the annulus was filled with water, and the sampleholding region was sealed with an insulated cover.

As soon as the sample was introduced onto the plate, the data were collected (time $t=0$ ).

The test methods employed were oscillatory temperature, time, stress, and frequency sweeps at a constant temperature of $37{ }^{\circ} \mathrm{C}$ and a physiological $\mathrm{pH}$ (except for the temperature sweep experiments, where the temperature was varied from 5 to $80^{\circ} \mathrm{C}$ and the $\mathrm{pH}=5$ was also tested).

A temperature sweep was used to study the thermal behavior of chitosan solutions with and without cross-linking agent at two different $\mathrm{pH}$ values. The experiments were performed at a low oscillation frequency $(1 \mathrm{~Hz})$ and a small deformation $(0.01)$ to ensure that the experimental conditions did not interfere with the gelation process. The chitosan solutions were subjected to a temperature ramp in the range of $5-80{ }^{\circ} \mathrm{C}$, with a rate of increase of $2{ }^{\circ} \mathrm{C} / \mathrm{min}$. The thermoreversibility of the gels was also investigated by decreasing the temperature from 80 to $5^{\circ} \mathrm{C}$ at the same rate $\left(2{ }^{\circ} \mathrm{C} / \mathrm{min}\right)$.

A time sweep at a constant shear frequency $(1 \mathrm{~Hz})$ in the linear viscoelastic region (LVR) was performed to monitor the in situ gelation behavior of the hydrogel dispersions, enabling the monitoring of the evolution of elastic stored modulus, $G^{\prime}$, and the viscous loss modulus, $G^{\prime \prime}$, with time. The dynamics of both viscoelastic characteristic functions were employed to determine the gelation time, the instant at which $G^{\prime}$ intersects $G^{\prime \prime}$. Alternatively, the gelation time was determined by employing the Winter-Chambon criterion, using multifrequency experiments in the range of 1 to $10 \mathrm{~Hz}$, aiming to validate the values achieved from time sweep tests at one frequency.

Finally, the stress sweep, at a constant frequency of $1 \mathrm{~Hz}$, was used on the hydrogels to determine the linear viscoelastic region profiles by shearing them until structure breakdown and to determine $G^{\prime}$ values. It should be noted that both the time and stress sweeps provide $G^{\prime}$ and $G^{\prime \prime}$ information on the structural integrity of the cross-linked network but in different physical environments. The hydrogels were also subjected to a frequency sweep in the linear viscoelastic region (stress of $20 \mathrm{~Pa}$ ) to study the viscoelastic performance over a wide range of frequencies $(0.01$ to $100 \mathrm{~Hz})$.

\section{Results and Discussion}

Oscillatory Temperature Sweep. In the range of temperatures studied, the pure chitosan solution, without any crosslinking agent, at $\mathrm{pH} 5$ did not turn into gel state when heated. On the contrary, the pure chitosan solution at the physiological $\mathrm{pH}$, became a gel as demonstrated by Figure 1. The sharp rise of the elastic stored modulus, $G^{\prime}$, upon heating (curve i), attributed to the gelation process, which indicates that the liquid solution turned into a solid-like gel in the vicinity of $50{ }^{\circ} \mathrm{C}$, while the decrease of the same modulus upon cooling (curve ii) reveals a certain thermoreversibility, that is, a tendency of the gel to return to the liquid state, as reported by other authors. As the temperature of the incipient gelation depends on the chitosan molecular weight and on the degree of deacetylation, ${ }^{6}$ the above value is specific to the sample under testing.

The gelation mechanism of gel systems prepared by neutralizing pure chitosan solutions with a weak base, such as $\beta$-glycerol-phosphate $\left(\mathrm{p} K_{\mathrm{a}}=6.65\right.$ at $\left.25{ }^{\circ} \mathrm{C}^{39}\right)$, may involve a broad range of molecular interactions, including electrostatic repulsion between like-charged chitosan chains, electrostatic attraction between the oppositely charged chitosan and the

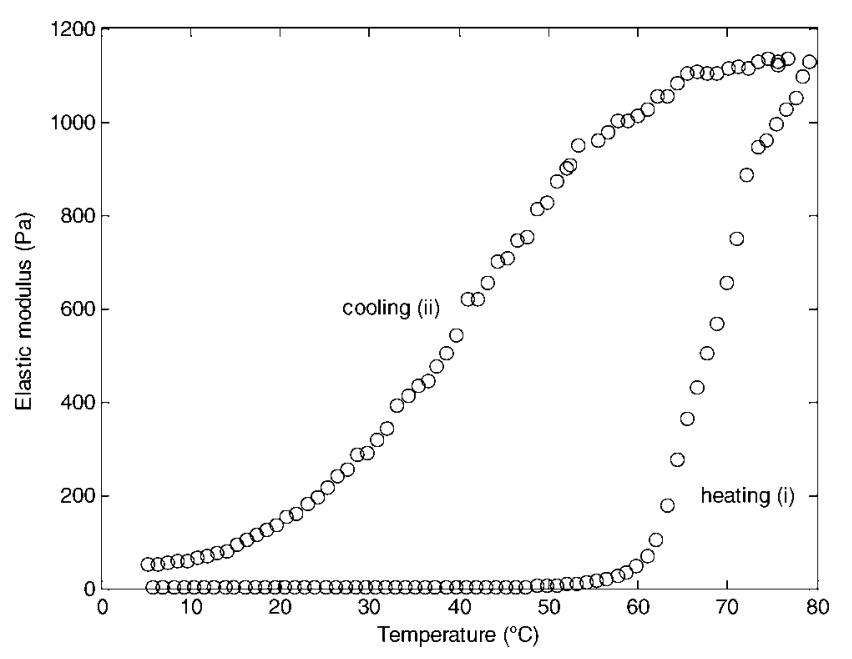

Figure 1. Sol-gel transition of a pure chitosan solution at the physiological $\mathrm{pH}(\mathrm{pH}=7)$ during a heating-cooling cycle between 5 and $80^{\circ} \mathrm{C}$, at $2^{\circ} \mathrm{C} / \mathrm{min}$.

Table 1. Dependence of the Temperature of Incipient Gelation on the $\mathrm{pH}$ Values and on the Cross-Linking Densities

\begin{tabular}{clc}
\hline & \multicolumn{2}{c}{ incipient gelation temperature ${ }^{a}\left[{ }^{\circ} \mathrm{C}\right]$} \\
\cline { 2 - 3 } genipin [\%w] & \multicolumn{1}{c}{$\mathrm{pH}=5$} & $\mathrm{pH}=7$ \\
\hline 0.00 & no gel formed & $47.4-48.5$ \\
0.05 & no gel formed & $45.3-46.4$ \\
0.10 & $69.6-70.7$ & $43.0-44.1$ \\
0.15 & $68.9-70.1$ & $37.5-38.6$ \\
0.20 & $65.2-66.3$ & $35.1-36.2$ \\
\hline
\end{tabular}

a Determined as the crossover point of $G^{\prime}$ and $G^{\prime \prime}$ curves.

phosphate moiety of $\beta$-glycerol-phosphate, hydrophobic effects, and hydrogen-bonding interactions between the chitosan chains. However, the hydrophobic interactions between the residual acetyl groups and the reduced solubility are the major driving forces for chitosan gelation at high temperature, in the presence of $\beta$-glycerol-phosphate. ${ }^{8}$

To investigate the gelation behavior of chitosan solutions with a cross-linking agent (genipin), chitosan solutions at different genipin loadings and $\mathrm{pH}$ values were subjected to temperature ramping. As expected, the cross-linking process revealed no thermoreversible characteristics. The results presented in Table 1 indicate that the temperature of incipient gelation, determined as the crossover point of the elastic $\left(G^{\prime}\right)$ and viscous $\left(G^{\prime \prime}\right)$ moduli, decreases both with the cross-linking density and with the $\mathrm{pH}$.

These results also show that, for chitosan solutions that have been neutralized to physiological $\mathrm{pH}$ via $\beta$-glycerol-phosphate, the addition of $0.15 \%$ of genipin provokes the transformation of the liquid solution into a solid-like gel at around $37^{\circ} \mathrm{C}$. Based on these observations and taking into consideration the in vivo applications of these gels (where the solution will be injected into the body and allowed to gel in situ), all subsequent rheological tests were performed at physiological conditions (a $\mathrm{pH}$ value of 7 and a temperature of $37^{\circ} \mathrm{C}$ ).

Oscillatory Time Sweep. To improve knowledge regarding the gelation kinetics and gel properties, the rheological behavior, decomposed in both components $G^{\prime}$ and $G^{\prime \prime}$ moduli, was monitored. Although the gelation time determination strategy, based on the intersection of elastic and viscous moduli, has been repeatedly used in literature, it often provides a rough approximation. ${ }^{28}$ To overcome this limitation, a more rigorous approach, based on multifrequency experiments (Winter-Chambon criterion) was also used. 

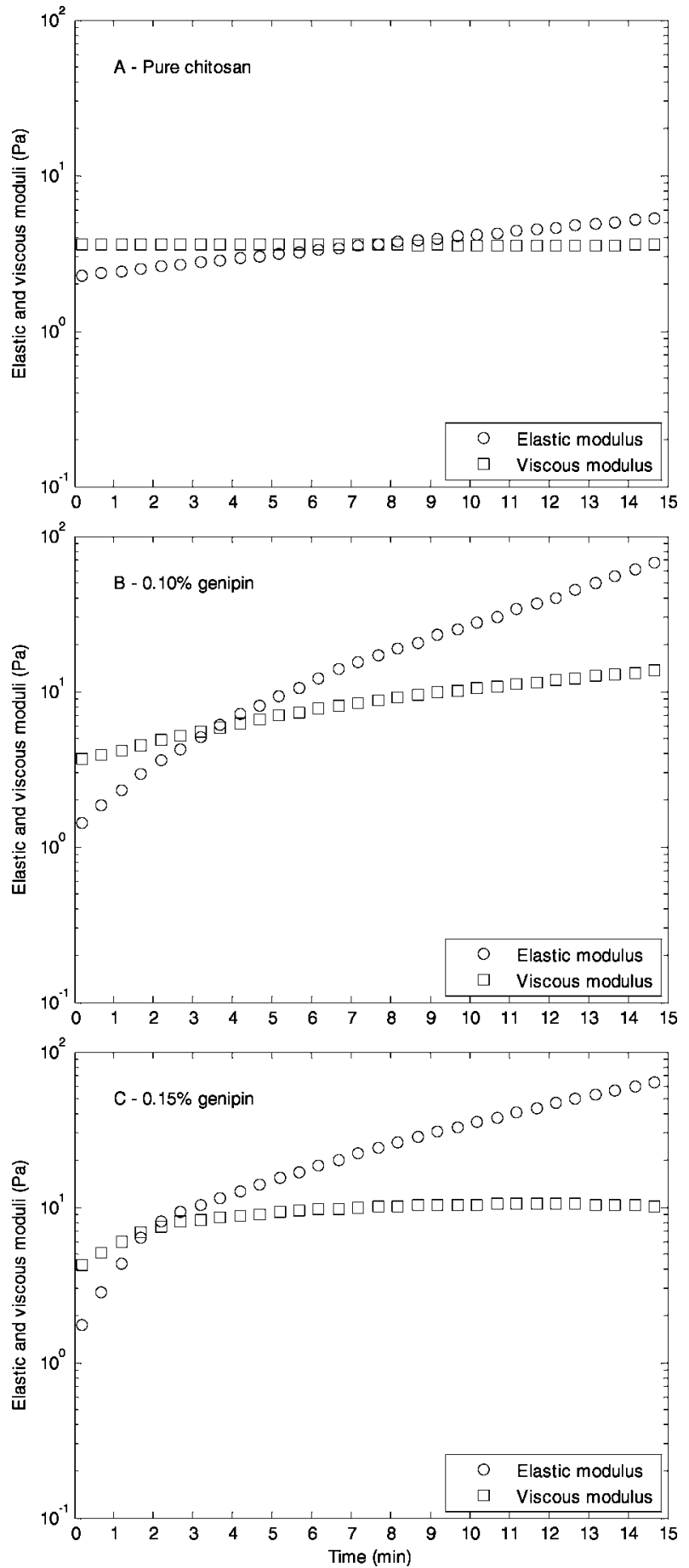

Figure 2. Dynamics of elastic, $G^{\prime}$, and viscous, $G^{\prime \prime}$, moduli near the gel point, at $1 \mathrm{~Hz}$ (A, pure chitosan; B and C, $0.10 \%$ (w) and $0.15 \%$ (w) genipin chitosan concentration network, respectively). The gelation time is determined as the time at which $G^{\prime}$ and $G^{\prime \prime}$ intersect each other.

Figure 2 shows the time sweep profiles of elastic $\left(G^{\prime}\right)$ and viscous $\left(G^{\prime \prime}\right)$ moduli near the gel point for pure chitosan solution (A) and for $0.10 \%(B)$ and $0.15 \%$ (C) genipin concentration networks. At the beginning, $G^{\prime \prime}$ was larger than $G^{\prime}$, which was expected because the samples were still in a liquid state and, thus, viscous properties dominated. As the solutions began to turn into a gel-like state due to the formation of the cross-linked network (in the case of networks containing $0.10 \%$ and $0.15 \%$ of genipin), both moduli increased. However, the rate of increase of $G^{\prime}\left(\Delta G^{\prime} / \Delta t\right)$ was higher than that of $G^{\prime \prime}$ because the elastic properties started to dominate. This difference in the rates leads
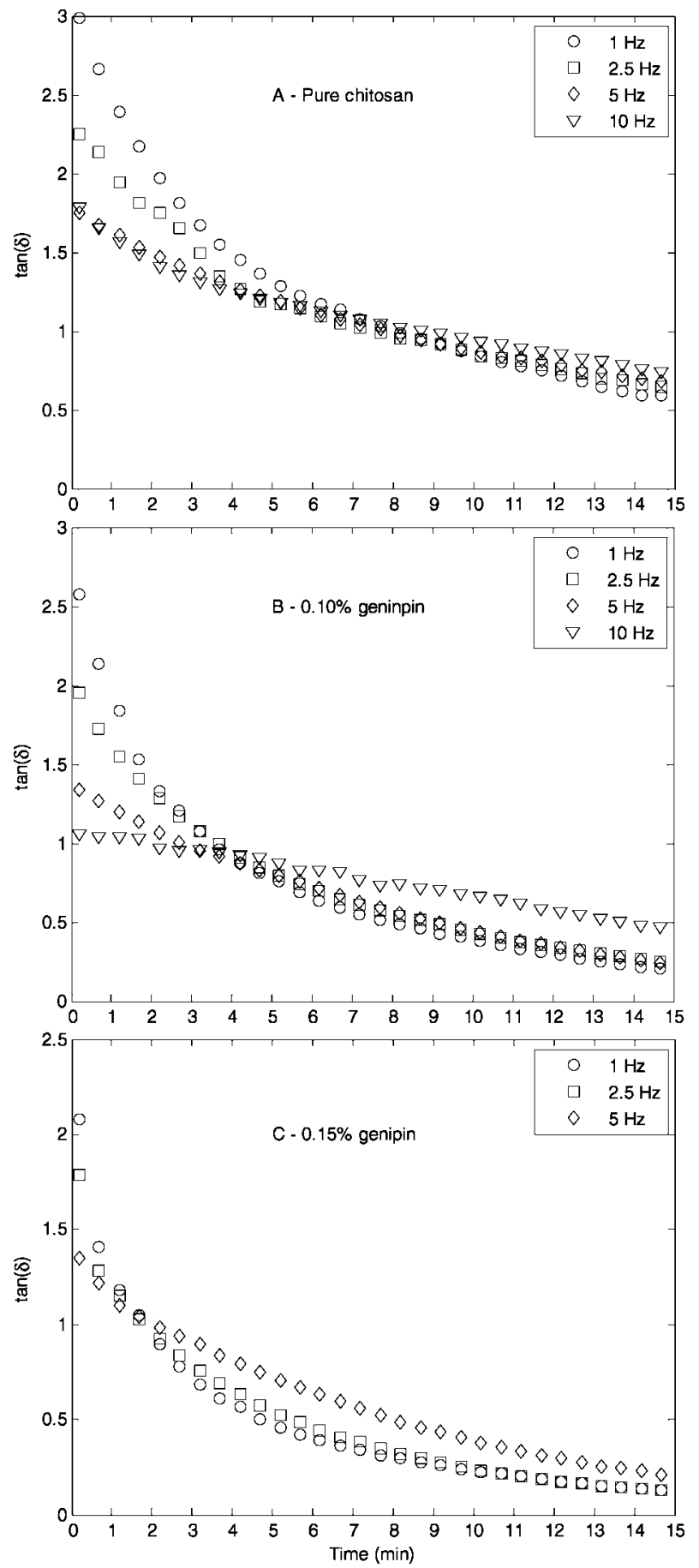

Figure 3. Loss tangent, $\tan (\delta)$ vs time for different constant shear frequencies (A, pure chitosan; B and C, $0.10 \%(w)$ and $0.15 \%(w)$ genipin chitosan concentration network, respectively). The gelation time is determined as the time at which $\tan (\delta)$ curves intersects.

to a $G^{\prime}$ and $G^{\prime \prime}$ crossover. The time required to achieve this crossover is, as mentioned above, the gelation time. From Figure 2 , it is also evident that higher genipin concentrations lead to lower gelation times, indicating that genipin is the rate limiting reactant during this early phase of the process. It should also be stressed that the gelation time decreases from about $8 \mathrm{~min}$ to about $2 \mathrm{~min}$ when the genipin concentration is increased from 0 to $0.15 \%$, which represents a significant reduction $(75 \%)$.

As pointed out before, the gelation time was also evaluated from the plots of loss tangent $(\tan (\delta))$ versus time at different constant frequencies for pure as well as cross-linked chitosan hydrogel networks. One can see from Figure 3 that $\tan (\delta)$ 
Table 2. Gelation Times and Relaxation Exponent Values for Chitosan Gels Cross-Linked with Different Genipin Concentrations

\begin{tabular}{ccc}
\hline $\begin{array}{c}\text { genipin } \\
{[\% \mathrm{w}]}\end{array}$ & $\begin{array}{c}\text { gelation } \\
\text { time }^{a}[\mathrm{~min}]\end{array}$ & $\begin{array}{c}\text { relaxation } \\
\text { exponent }^{b}\end{array}$ \\
\hline 0.00 & 8.19 & $0.63 \pm 0.02$ \\
0.10 & 3.68 & $0.61 \pm 0.02$ \\
0.15 & 1.68 & $0.66 \pm 0.01$ \\
\hline
\end{tabular}

${ }^{a}$ Defined as the time at which $\tan (\delta)$ is independent of the frequency. ${ }^{b}$ Calculated from eq 5: $n=2 / \pi \tan ^{-1}(\tan (\delta))$ at the gel point.

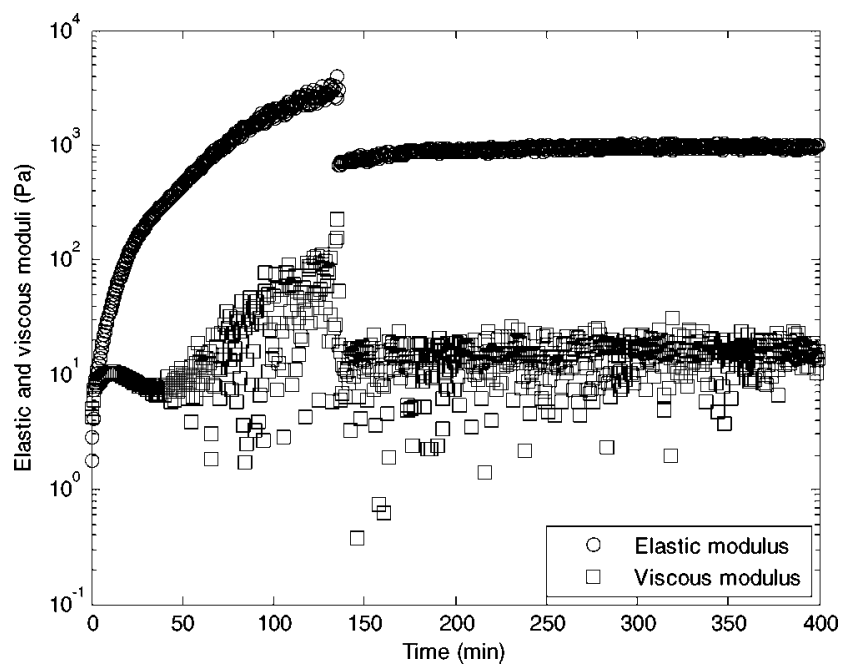

Figure 4. Dynamics of elastic and viscous moduli for a chitosan network cross-linked with $0.15 \%(w)$ genipin.

becomes frequency independent at the gel point. Beyond this point, $\tan (\delta)$ decreases more gradually with time due to the fact the magnitude of the rise of $G^{\prime}$ is higher than that of $G^{\prime \prime}$ as a result of the formation of an elastic fractal gel. As indicated in Table 2, the formulations studied have reached the gel point for different gelation times, these being very similar to those achieved by the first approach (Figure 2). These results show that the gelation time is, as expected, inversely proportional to the genipin concentration. The cross-linking density dependence of the gelation is well described $\left(R^{2}=0.97\right)$ by

$$
t_{g}=8.65 e^{-10.19 C}
$$

where $t_{\mathrm{g}}$, is the gelation time ( $\left.\mathrm{min}\right)$, and $C$ the genipin loading.

Together with the gelation times, Table 2 also lists the values of the relaxation exponent, $n$, estimated from the values of $\tan (\delta)$ at the gel point (Figure 3) using eq 5. The values of the relaxation exponent at the gel point $(\approx 0.63)$ are in good agreement with the value predicted from the percolation theory $(n \approx 2 / 3)^{37}$

It can be concluded that for applications that require chitosanbased matrices with reduced gelation times, genipin is a potentially useful cross-linking agent. In fact, long gelation times can lead, for example, to excessive drug diffusion in drug delivery systems ${ }^{40}$ or heterogeneous cell distribution within the matrix as in tissue engineering. ${ }^{23}$ Genipin can thus be used in optimally designing the gel with respect to the gelation time.

To investigate the gelation mechanism within a larger period of the time, the viscoelastic properties of solutions during the gelling process (without and with cross-linking) were monitored by performing time sweep experiments. Figure 4 shows the typical time sweep profiles of $G^{\prime}$ and $G^{\prime \prime}$ for a chitosan network that was cross-linked with $0.15 \%$ genipin (up to $7 \mathrm{~h}$ ).

The most striking feature of this figure is the presence of a discontinuity in the dynamics of $G^{\prime}$. In fact, this curve shows that the cross-linked chitosan hydrogel reaches an upper strength limit, followed by a faster reduction, with the form of a near discontinuity at around $130 \mathrm{~min}$, subsequently achieving the structural network steady state.

Similar profiles, obtained with an analogous rheometer, have been reported by other authors for different systems (aqueous chitosan-2,5-dimethoxy-2,5-dihydrofuran ${ }^{41}$ and organogels produced from poly(ethylene glycol) and dibenzylidene sorbitol). ${ }^{42}$ The sudden reduction of the elastic modulus is probably related to the syneresis properties of the cross-linked gels. Typically observed in strong polysaccharide gels, including some crosslinked chitosan systems, syneresis is a process that occurs when the cross-link density reaches a critical point at which the network pressure exceeds the osmotic counter-pressure that is generated by water bound within the gel structure. ${ }^{41}$ As a result, the gelled body contracts in size, expelling free water (this was experimentally observed during the preparation of the cured hydrogels). Syneresis is characterized by a molecular reorganization of the macromolecular network involving both the disruption and the formation of some bonds. ${ }^{43}$ When occurring during cone-and-plate oscillatory rheometry, syneresis can affect experimental measurements by causing the gel to shrink away from the cone. Such behavior leads to a large reduction in the contact area between the gel and the rheometer cone, making $G^{\prime}$ reach a maximum value over a relative short time frame, beyond which its values rapidly decrease.

In addition to the peculiar $G^{\prime}$ behavior, Figure 4 also shows the random variation of $G^{\prime \prime}$, which is due to the values of the angle $\delta$ being below the sensitivity limit of the transducer of the rheometer. Nevertheless, these values are always larger than zero, indicating the viscoelastic nature of these hydrogels.

Oscillatory Stress Sweep. The stress range over which $G^{\prime}$ is independent of the applied shear stress is the linear viscoelastic region (LVR). The LVR profiles of all cross-linked hydrogel networks were determined, as well as that of pure chitosan hydrogel, by subjecting them to a stress sweep until structure breakdown. Because the cross-linking reaction is expected to develop over varying time scales (minutes to days), the evaluation of rheological tests with time becomes impractical. In the present work, the rheological properties, namely, the LVR profile and frequency response of $G^{\prime}$, were determined considering that the fixed curing time is $12 \mathrm{~h}$.

The effect of the stress amplitude on the cured hydrogels is shown in Figure 5. Over the LVR and as indicated in the subset graph on the left, the applied stress is in phase with the resulting strain $(\delta=0)$. Beyond the LVR, the elastic modulus abruptly decreased, indicating that the structure breakdown occurred as a consequence of the large deformations imposed (stress is not in phase with the corresponding strain $(\delta>0)$, as depicted in the subset graph on the right). The stress at which a polymeric system begins to show nonlinear viscoelastic behavior is generally designated as the critical shear stress.

Figure 5 also shows that the augmentation of the cross-linking density leads to $(i)$ an expected increase of $G^{\prime}$, because it represents the elastic component of the materials deformation which, in turn, is correlated with the number of effective intermolecular cross-links formed in the hydrogel network and (ii) an increase in the critical shear stress and, consequently, the extension of LVR. Indeed, the critical shear stress of the highest cross-linking concentration network overcomes the pure chitosan network shear stress by 1 order of magnitude $(600 \mathrm{~Pa}$ for a $0.20 \%$ genipin network against $60 \mathrm{~Pa}$ for the pure chitosan hydrogel). 


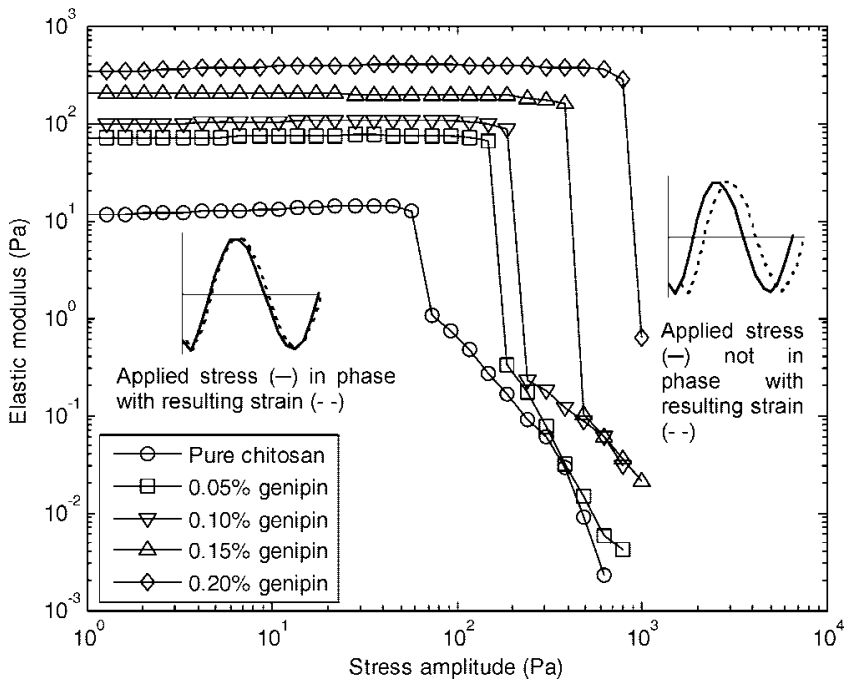

Figure 5. Linear viscoelastic region (LVR) profiles of $12 \mathrm{~h}$-cured, cross-linked, and pure chitosan hydrogels.

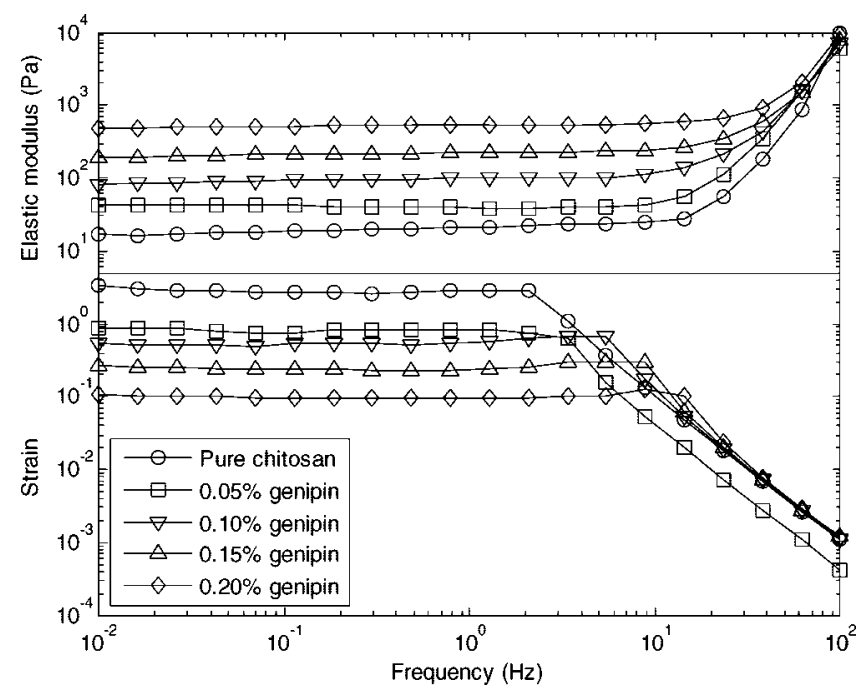

Figure 6. Evolution of elastic modulus and strain as a function of the applied frequency of oscillatory shear stress.

Oscillatory Frequency Sweep. Frequency sweep experiments were carried out to investigate the gel properties of the cured systems, namely, the stability of three-dimensional cross-linked networks. The cured hydrogels were subjected to a frequency sweep from 0.01 to $100 \mathrm{~Hz}$. From the LVR profiles presented above (Figure 5), an appropriate shear stress (20 Pa) was selected to be as high as possible to avoid a torque that was too low.

Figure 6 presents the plot of $G^{\prime}$ versus oscillatory frequency (top panel). The data obtained reveal that for all of the hydrogels, $G^{\prime}$, exhibits a plateau in the range $0.01-10 \mathrm{~Hz}$, which is indicative of a stable cross-linked network. This $G^{\prime}$ frequencyindependent feature also indicates a solid-like behavior in the cured systems. At higher frequencies, all of the hydrogels presented an increase in $G^{\prime}$, corresponding to the highest rate of increase with respect to the pure chitosan hydrogel and the lowest with respect to the hydrogel with largest percentage of genipin $(0.20 \%)$.

This behavior was expected because the magnitude of the viscoelastic response of a polymeric network is first governed by the length of the flexible polymer chains and then by the nature of the imposed mechanical motion. ${ }^{44-46}$ Longer chains have characteristic longer relaxation times. The polymer chain segments between cross-links are longer for the less cross-linked networks and, thus, will give origin to lower molecular motion frequencies than those arising from the highly cross-linked networks. However, at higher frequencies, long chains fail to rearrange themselves in the time scale of the imposed motion and, therefore, stiffen up, assuming a more "solid-like" behavior that is characterized by a sharp increase in $G^{\prime}$, as observed in Figure 6, because shorter polymer chains resulting from highly cross-linked networks exhibit smaller relaxation times. They require higher applied frequencies to obtain a similar response, leading to a gradual rise in $G^{\prime}$.

The plot of strain versus applied frequency (bottom panel in Figure 6) further emphasizes this behavior. Indeed, at lower frequencies (up to $1 \mathrm{~Hz}$ ), the hydrogel network that is crosslinked with the highest genipin concentration exhibits a smaller strain compared to that of the hydrogel cross-linked with $0.05 \%$ of genipin because the former is stiffer, as it was shown in Figure 5. However, due to chain stiffening at higher frequencies, hydrogels obtained with a genipin concentration of $0.05 \%$ produce less deformation than the highly cross-linked networks, thus leading to a more "solid-like" structure.

These results show that the genipin reagent can be used to provide cross-linking chitosan networks whose mechanical performance can be tailored to meet the demands of specific applications.

\section{Conclusions}

Rheometry was used to elucidate the formation and the final structure of hydrogels that were prepared by the interaction/ reaction of chitosan and genipin as the cross-linker. The effect of the genipin loading on the network was also evaluated. Rheological monitoring during hydrogel synthesis was employed to determine the gelation time, a determinant variable in the use of biopolymeric structures for injectable or scaffold ends.

The gelation time was measured by two different rheological approaches: (i) by the crossover point of the $G^{\prime}$ and $G^{\prime \prime}$ and (ii) by the Winter-Chambon criterion. Both approaches provide consistent estimates and the results are in excellent agreement. At $37^{\circ} \mathrm{C}$ and physiological $\mathrm{pH}$, the results showed that the crosslinking biopolymer genipin at a loading of $0.15 \%$ reduces the gelation time from $8 \mathrm{~min}$ (corresponding to pure chitosan) to less than $2 \mathrm{~min}$, which represents an improvement of around $75 \%$.

The results also showed that the increase of the cross-linker loading in the cured hydrogel led to an increase of the steady state $G^{\prime}$ and, thus, the enhancement of their elasticity, which is a clear indication of the improvement on the three-dimensional cross-linked networks stability.

The main conclusion of this study is that, under physiological conditions, the viscoelastic features of a chitosan solution, and its gelling ability, can be tuned by changing the genipin loading. In view of this behavior, these formulations can be produced in situ. They are, thus, promising matrices for the encapsulation cells and bioactive molecules. However, the biological performance of these formulations should be evaluated before final conclusions can be drawn.

Acknowledgement. The authors would like to thank Department of Ceramics and Glass Engineering from University of Aveiro for providing direct access to the C-VOR rheometer.

\section{References and Notes}

(1) Ravi, M. N. V. K. React. Funct. Polym. 2000, 46, 1-27.

(2) Yi, H.; Wu, L.-Q.; Bentley, W. E.; Ghodssi, R.; Rubloff, G. W.; Culver, J. N.; Payne, G. F. Biomacromolecules 2005, 6, 2881-2894. 
(3) Ravi, M. N. V. K.; Muzzarelli, R. A. A.; Muzzarelli, C.; Sashiwa, H.; Domb, A. J. Chem. Rev. 2004, 104, 6017-6084.

(4) Rinaudo, M.; Auzely, R.; Vallin, C.; Mullagaliev, I. Biomacromolecules 2005, 6, 2396-2407.

(5) Jackson, D. S. U.S. Patent 4,659,700, 1987.

(6) Chenite, A.; Chaput, C.; Wang, D.; Combes, C.; Buschmann, M. D.; Hoemann, C. D.; Leroux, J. C.; Atkinson, B. L.; Binette, F.; Selmani, A. Biomaterials 2000, 21, 2155-2161.

(7) Chenite, A.; Buschmann, M.; Wang, D.; Chaput, C.; Kandani, N. Carbohydr. Polym. 2001, 46, 39-47.

(8) Cho, J.; Heuzey, M. C.; Bégin, A.; Carreau, P. J. Biomacromolecules 2005, 6, 3267-3275.

(9) Khurma, J. R.; Rohindra, D. R.; Nand, A. V. Polym. Bull. 2005, 54, 195-204.

(10) Francis, S. J. K.; Mattew, H. W. T. Biomaterials 2000, 21, 25892598.

(11) Alexeev, V. L.; Keberg, E. A.; Evmenenko, G. A. Polym. Eng. Sci. 2000, 40, 1211-1215.

(12) Budtova, T.; Belnikevich, N.; Kalyuzhnaya, L.; Alexeev, V.; Bronnikov, S.; Vesnebolotskaya, S.; Zoolshoev, Z. J. Appl. Polym. Sci. 2002, 84, 1114-1122.

(13) Mi, F. L.; Tan, Y. C.; Liang, H. C.; Huang, R. N.; Sung, H. W J. Biomater. Sci. Polym. Ed. 2001, 12, 835-850.

(14) Jin, J.; Song, M.; Hourston, D. J. Biomacromolecules 2004, 5, 162 168.

(15) Lin, C. C.; Metters, A. T. Adv. Drug Delivery Rev. 2006, 58, 13791408 .

(16) Balakrishnan, B.; Jayakrishnan, A. Biomaterials 2005, 26, 3941-3951.

(17) Sung, H. W.; Liang, I. L.; Chen, C. N.; Huang, R. N.; Liang, H. F. J. Biomed. Mater. Res. 2001, 55, 538-546.

(18) Bigi, A.; Cojazzi, G.; Panzavolta, S.; Roveri, N.; Rubini, K. Biomaterials 2002, 23, 4827-4832.

(19) Mi, F. L.; Shyu, S. S.; Peng, C. K. J. Polym. Sci., Part A: Polym. Chem. 2005, 43, 1985-2000.

(20) Mi, F. L.; Sung, H. W; Shyu, S. S. J. Polym. Sci., Part A: Polym. Chem. 2000, 38, 2804-2814.

(21) Sung, H. W.; Huang, R. N.; Huang, L. L.; Tsai, C. C. J. Biomater Sci. Polym. Ed. 1999, 10, 63-78.

(22) Bhattarai, N.; Ramay, H. R.; Gunn, J.; Matsen, F. A.; Zhang, M. J. Controlled Release 2005, 103, 609-624.

(23) Hou, Q.; De Bank, P. A.; Shakesheff, K. M. J. Mater. Chem. 2004, 14, 1915-1923.
(24) Rwei, S. P.; Chen, T. Y.; Cheng, Y. Y. J. Biomater. Sci. Polym. Ed. 2005, 16, 1433-1445.

(25) Hwang, J. K.; Shin, H. H. Korea-Aust. Rheol. J. 2000, 12, 175-179.

(26) Montembault, A.; Viton, C.; Domard, A. Biomaterials 2005, 26, 1633 1643.

(27) Shon, S. O.; Ji, B. C.; Han, Y.; A; Park, D. J.; Kim, I. S.; Choi, J. H J. Appl. Polym. Sci. 2007, 104, 1408-1414.

(28) Montembault, A.; Viton, C.; Domard, A. Biomaterials 2005, 6, 653662 .

(29) Gibson, S. L.; Walls, H. J.; Kennedy, S. B.; Welsh, E. R. Carbohydr. Polym. 2003, 54, 193-199.

(30) Butler, M. F.; Yiu-Fai, N. G.; Pudney, P. D. A. J. Polym. Sci., Part A: Polym. Chem. 2003, 41, 3941-3953.

(31) Pai, V.; Srinivasarao, M.; Khan, S. A. Macromolecules 2002, 35, 1699_ 1707.

(32) Mezger, T. G. The Rheology Handbook; Zorll U., Ed.: Verlag: Germany, 2002.

(33) Zao, Y.; Cao, Y.; Yang, Y.; Wu, C. Macromolecules 2003, 36, 855859.

(34) Matricardi, P.; Dentini, M.; Crescenzi, V. Macromolecules 1993, 26, 4386-4387.

(35) Grisel, M.; Muller, G. Macromolecules 1998, 31, 4277-4281.

(36) Winter, H. H.; Chambon, F. J. Rheol. 1986, 30, 367-382.

(37) Scanlan, J. C.; Winter, H. H. Macromolecules 1991, 24, 47-54.

(38) Madbouly, S. A.; Otaigbe, J. U. Macromolecules 2005, 38, 1017810184.

(39) Alberty, R. A. Physical chemistry, 6th ed.; John Wiley \& Sons: Toronto, 1983.

(40) Hafeti, A.; Amsden, B. J. Controlled Release 2002, 80, 9-28.

(41) Johnson, S. B.; Dustan, D. E.; Franks, G. V. Colloid Polym. Sci. 2004, 282, 602-612.

(42) Wilder, E. A.; Hall, C. K.; Khan, S. A.; Spontak, R. J. Langmuir 2003, 19, 6004-6013.

(43) Vachoud, L.; Zydowicz, N.; Domard, A. Carbohydr. Res. 2000, 326, 295-304.

(44) Anseth, K. S.; Bowman, C. N.; Brannon-Peppas, L. Biomaterials 1996, 17, 1647-1657.

(45) Kavanagh, G. M.; Ross-Murphy, S. B. Prog. Polym. Sci. 1998, 23, 533-562.

(46) Ghosh, K.; Shu, X. Z.; Mou, R.; Lombardi, J.; Prestwich, G. D.; Rafailovich, M. H.; Clark, R. A. F. Biomacromolecules 2005, 6, 2857-2865.

\section{BM700762W}

